Check for updates

London, UK

Cite this as: $B M J$ 2021;372:n778 http://dx.doi.org/10.1136/bm..n778 Published: 19 March 2021

\title{
Covid-19: Eight in 10 Wuhan residents with antibodies had no symptoms, finds study
}

\section{Susan Mayor}

More than three quarters of Wuhan residents who tested positive for SARS-CoV-2 antibodies had experienced no covid-19 symptoms and just under half developed long lasting neutralising antibodies, show results from the first long term seroprevalence study from the epicentre of the outbreak in China. ${ }^{1}$

Researchers tested 9542 people from 3556 randomly selected households across the 13 districts of Wuhan, which has a total population of 11 million. Of these, 532 people (5.6\%) tested positive for

pan-immunoglobulins against SARS-CoV-2 when the city's lockdown ended in April 2020, giving an overall population seroprevalence of $6.92 \%$ ( $95 \%$ confidence interval 6.41 to 7.43 ). Seroprevalence was higher in women, people aged 66 and over, and in healthcare workers.

Just over $80 \%$ of people testing positive for SARS-CoV-2 antibodies (437 of 532 participants) reported having no covid-19-related symptoms, showed results reported in the Lancet.

"The proportion of asymptomatic people was much higher than the average proportions of $40-45 \%$ that have been reported worldwide," said the researchers, led by Chen Wang, from the Chinese Academy of Medical Sciences and Peking Union Medical College, China. He acknowledged that there may have been recall bias but noted that stringent measures had been taken to identify covid-19 cases and encourage Wuhan residents to record symptoms during the outbreak.

"Given that asymptomatic people are not usually screened, there may be large discrepancies between reported covid-19 cases and actual infected cases, which has been proven by the experiences and data from other countries," suggested Wang.

Follow-up testing showed that $40 \%$ of people with antibodies against SARS-CoV-2 (212 of 532 ) developed neutralising antibodies, giving protection against future infection. These remained for at least nine months regardless of whether they had symptoms or not, although levels were higher in those with confirmed disease or symptoms.

"Efficient global management of covid-19 will probably succeed or fail on the basis of immunity induced by natural infection and, especially, vaccination. Given the relative paucity of neutralising antibodies through natural infection, the study reinforces the need for effective covid-19 vaccines in the population level control of the disease," said Richard Strugnell and Nancy Wang, from the Peter Doherty Institute at the University of Melbourne, Australia, in a linked commentary. ${ }^{2}$
They added, "The study is an important milestone in the description of SARS-CoV-2 infection and our understanding of immunity in the pandemic. It provides a deeper understanding of natural seroconversion in a key city in the pandemic and the findings underscore the remarkable achievement of the Chinese public health system in controlling the Wuhan outbreak of covid-19 at a time when testing, tracing, and treatment resources were much less developed."

He Z, Ren L, Yang Y, et al. Seroprevalence and humoral immune durability of anti-SARS-CoV-2 antibodies in Wuhan, China: a longitudinal, population-level, cross-sectional study. Lancet 2021;397:1075-84.

2 Strugnell R, Wang N. Sustained neutralising antibodies in the Wuhan population suggest durable protection against SARS-CoV-2. Lancet 2021;397:1037-9.

This article is made freely available for use in accordance with BMJ's website terms and conditions for the duration of the covid-19 pandemic or until otherwise determined by BMJ. You may use, download and print the article for any lawful, non-commercial purpose (including text and data mining) provided that all copyright notices and trade marks are retained. 